\title{
FEDERATED HBIM MODELS FOR CULTURAL HERITAGE: SURVEY MODEL AND CONCEPTUAL MODEL
}

\author{
D. Delpozzo ${ }^{1}$, L. Appolonia ${ }^{2}$, B. Scala ${ }^{3}$, A. Adami ${ }^{1}$ \\ ${ }^{1}$ He.Su.Tech. group, MantovaLab, Dept. of Architecture, Built environment and Construction engineering (ABC), Politecnico di \\ Milano, Piazza d'Arco, 3, 46100 Mantua, Italy, damiano.delpozzo@mail.polimi.it, andrea.adami@polimi.it \\ ${ }^{2}$ RAVA, Piazza Severino Caveri, 11100 Aosta, Italy. l.appolonia@regione.vda.it \\ ${ }^{3}$ DICATAM, Università degli studi di Brescia, Via Branze 43, 25123 Brescia, Italy. barbara.scala@unibs.it
}

\section{Commission II}

KEY WORDS: HBIM, geometric modelling, Cultural Heritage, point cloud, federated models

\begin{abstract}
:
The federation of models is a pillar of the BIM (Building Information Modelling) approach: it allows to keep the contents of each discipline separate during the modelling-creation phase, and to merge them together later during the project management phase, from its feasibility to the construction site, to the management of the entire life-cycle of the building.

Generally, these models refer to specific disciplines and the architectural, plant and structural model are always identified.When the asset belongs to Cultural Heritage, more generically an existing building, the BIM approach (at this point HBIM - Historic Building Information Modelling) is faced with an additional level of complexity since it is necessary to model something existing (the building in fact) and of which not much information is known. The geometric complexity of the asset often aggravates this situation because if the parametric modelling is preferred, it is difficult to represent such irregular morphologies, and if the surface modelling and a more geometrical detail is preferred, the model becomes very heavy.In many cases the choice is to approximate reality as best as possible through specific and tailor-made modelling approaches, often complex and with some borderline methods, if compared to BIM logic. In other cases, however, it makes sense to define when the geometric complexity and the reliability of the model are necessary, and when, instead, a simplification is required in order to effectively manage the information.

The case study presented in this paper is the Arch of Augustus, in Aosta, for the HBIM approach it has been chosen to separate the two approaches,placing side by side the federated models referred to the classical disciplines with two models of the current state: one very accurate with the purpose of maintaining all the quality of acquired 3D geometric information, while the other absolutely schematic, necessary as a $3 \mathrm{D}$ index for the information.

The approach described here, however, requires a preliminary reflection in order to define the BIM granularity - the smallest object in the model - and to define the methodological procedures that allow the bidirectional relationship between survey model and conceptual model. This paper provides insigth oif the importance of the relation between survey model and conseptual model. Future works will try to make this relation more stronger and efficient.
\end{abstract}

\section{INTRODUCTION}

Much research on Historic Building Information Modelling (HBIM) demonstrated the effectiveness of this approach in the management of the information content related to the conservation and restoration project. It seems clear, therefore, the need and the opportunity to go down this road to identify, with greater precision, which are the application areas for which the HBIM system is ready and which, instead, still require a phase of in-depth study and research.

The research has also highlighted how some phases are still very complex and often require a non-standard approach, an attitude that somehow collides with the very nature of BIM (Building Information Modelling. Among the activities that still present some difficulties, there is certainly the management of geometry in an HBIM model through commercial software.

Numerous studies and experiences have described the interoperability of software and therefore suggested the geometric modelling in software environments (Rhinoceros, Freecad, Sketchup), different from those of BIM Authoring. This is an attempt to compensate for the obvious limitations of commercial suites when modeling complex objects (in this case the Autodesk BIM suite, with Revit, Dynamo, and other applications), by performing modelling in contexts that allow greater geometric control. This approach is certainly workable and motivating, but it presents some problems when trying to model not only geometric content but also informative content.
The 3D geometric shape is correct, but it is not so easy to manage the information that should be linked to it.

A different approach, that finds however its origin in the same concept of federated model that characterizes the BIM systems, is the one that foresees the construction of more models, each one with its own purpose.

In literature, the discipline itself characterizes the single model, so there is a structural model or a plant model, referring respectively to structures and plants. It is possible, however, to think of developing other models; there are models which describes the historical development of an architetectural complex, and this is a reading of the heritage that, in manu cases, is fundamental for the preservation.

The proposal, here, is to have two models more than the traditional ones (architectural, structural and mechanicalelectrical-plumbing MEP) concerning the current situation: one is the survey model, very accurate and reliable, useful to extract geometric and dimensional information, the other could be called a conceptual model and it plays as the index for very easy and efficient management of information.

The simultaneous use of these two models requires to choose, time by time, the source of needed information, but, moreover, it requires to clearly define which are the goals of each model, its use and characteristics. For each model, it is indispensable to define the type of geometric model to use (made by points, by surfaces, or solid, parametric or not, which kind of surfaces, 
etc). But also, especially with the goal of managing information, which is the smallest element of the model, its granularity, where to link all informative contents.

The paper presents in chapter 2 some related works to the themes of modelling and managing complex architectures and shapes. In the following part, after the description of the methodology borrowed from the typical BIM approach, the goals and characteristics of each model are described. In the last part, after a short description of the study case of the Arch of Augustus in Aosta, the results are described and discussed, to evaluate the effectiveness of the method.

\section{RELATED WORKS}

The main concept of the method refers to federated models. They are a group of different systems, connected in a single entity. If considered independently each model represents only some features of the building and, moreover, all related to the same discipline.

The definition in the Associated General Contractors (AGC) ConcensusDocs 301- BIM Addendum of the federated model is particularly clear: a model consisting of linked but distinct component models, drawings derived from the models, texts, and other data sources that do not lose their identity or integrity by being so linked, so that a change to one component model in a federated model does not create a change in another component model in that federated model. A single federated model is useful for design co-ordination, clash avoidance and clash detection, approvals processes, design development, estimating and so on, but the individual models do not interact, they have clear authorship and remain separate.

The approach of federated models is not completely new for Cultural Heritage, as evident in (Solihil et al., 2016) where the authors deal with the purpose of a IFC (Industry Foundation Classes) approach to foster the connection and interoperability between the different models.

But in the context of this research about Cultural Heritage and their management for conservation, it is very important to reflect on the previous definition. Considering the legislative and contractual purposes of the AGC, that definition is particularly relevant because it also incorporates the concept of data authorship/responsibility which is independent of the use made of the model. In the field of Cultural Heritage, and consequently, of HBIM approach, many disciplines are involved, each one with its own peculiarity and role. According to this principle of federated models, each discipline has a specific role, and it is responsible for that.

In this case, Geomatics has two different tasks: to provide the metric data and to allow the management and geo-referencing of the data that any other discipline can provide. This duplex role of Geomatics deals with the complexity of HBIM data processing which is, even if not exhaustive, $3 \mathrm{~d}$ modelling and segmentation. They are the logical steps of the Scan2BIM process where the quality of the measurements, if done accurately, is generally not questioned, whereas it is always necessary to consider the quality of the transformation from points (point cloud) to 3D model (surfaces, parametric, etc.) and the subdivision of the entire complex into smaller objects.

In this way, once the choice has been made to work in the HBIM field with two different models, both related to geomatics by genesis and responsibility, we fall back on the theme of the Scan2BIM process which, according to the authors (Adami, 2021), is still far from being optimized and above all made automatic due to the complexity, irregularity and uniqueness that characterize Cultural Heritage.

However, the same doubts and possibilities arise as those highlighted in the extensive bibliography on the Scan2Bim process. The main doubts concern the choice of modelling principle between parametric, polygonal surfaces and curved surfaces.

The literature in the field is very deep and rich both for the state of art both for suggestion and proposals according to different study-cases and applications as evident in bibliographic research using the keywords "Scan-to-BIM". According to the different topics some recent papers can be cited such as (Yang et al., 2020) (Costantino et al., 2021) (Santagati et al., 2021).

Some papers are more specific and they deal with suggestions to overcome the problem of modelling, i.e. (Brumana et al., 2020) (Banfi 2019) (Barazzetti, 2016).

\section{METHODOLOGY}

Starting from the idea of a federated model, the two proposals that are considered in this research represent the reality-based model (survey model) and an ideal representation of the same entity, defined as conceptual model. The difference is not in the content, as it happens between architecture, structure and MEP model, but it is the nature itself of the model and its role.

In concrete terms, the models are the survey model, a purely geometric, characterized by high metric accuracy and reliability (guaranteed through the control of the methods of survey and modelling), and the conceptual model which aims to bring all the information to the ideal, original structure of the building.

This difference is very evident both in the choices of different types of 3D modelling and in the granularity of the model.

The survey model, which has to be very accurate, precise and reliable, could be built as a point model (pointcloud) or a mesh. The second approach, based on surfaces, is more mimetic of reality but it is also more complex to be managed in a BIM authoring system in terms of the number of faces and file dimension.

The first one is more abstract (it is possible to see through the object) but it is already implemented in the BIM process. Point clouds are nowadays very easy to import and manage even in commercial software. Considering that from the survey model the queries are concerning shapes and dimensions, a point cloud replica, made by reality-based system, can be considered to all intents and purposes a digital $3 \mathrm{~d}$ replica of real architecture.

The other model, conceptual, has to be, instead, very easy to use and to be managed and, as already said, not so related to real objects. To do it, and to follow the principles of BIM modelling, the parametric approach is the most suitable to build similar objects. Dealing with the approach of the most common commercial BIM authoring software, it means to define some families of objects and develop many other instances.

Of course, to allow the bi-directional link between the two models, they have - to share the same reference system. It may seem trivial to specify this concept in this context, as it is a founding element of the principle of federated models. But it is important to reiterate it, instead, in a field that derives from geomatics. It is also important to know how modeling how modelling software handles models and coordinates. In fact, they are not designed to manage large coordinates (georeferenced data) and therefore perform automatic translations between the topographical reference system chosen for the survey (both local or absolute) and the internal one of the model. Typically, this means having dual points of origin, some in the topographic system and others in the model's internal system. 
If the modelling phase is developed correctly, not only in terms of geometric commands, but also in terms of georeferencing the data, the two models can coexist, and we will turn to a model or the other depending on the needs. Both georeferenced in the same reference system, they will be three-dimensional indexes of different contents: only geometric data in a model (survey) and informative, at multiple levels, in the model that can be defined as conceptual. Such an approach allows also to make up for the limits still evident in the modelling of complex forms.

The last characteristic to be set/defined for each model is its granularity. In the survey model, the smallest element is defined according to the digital nature of the file itself: single point of the pointcloud or single face of a mesh. It is not really a semantic subdivision of the model, but it is not strictly necessary as the role of this model is to contain metrical information.

On the other hand, instead, it is very fundamental for the conceptual model to define its smallest objects. In fact, they represent the element where all the information is linked. In many cases the technological elements could be a very interesting choice, especially dealing with restoration. In some other cases, more related to material conservation and preservation, this choice is more debated.

\subsection{The case study: the Arch of Augustus in Aosta}

This approach has been successfully tested for the Arch of Augustus in Aosta. Of course, the monument itself is not enough to define the characteristics and goals of the BIM model.

The arch, a symbol of the city, as well as an element characterizing the access to the historic centre, needs conservation works, seizing this opportunity it was decided to develop a conservation project with BIM mode (Adami et al., 2021).

The Arch of Augustus in Aosta was built contemporary to the foundation of the city, about in the 23-25 a.C: it is an honorary arch since triumphal arch could be built only in Rome. It commemorates Augusto's victories over the Salassi Celtic tribe. The arch was not inside the city, it was built right after the bridge on Buthier creek, the road crossed under the vault of the arch, leading to the main city gate: Porta Praetoria. It has one fornice, with four Corinthian columns on the main façade, and three on the lateral, the entablature had a Doric frieze with triglyph and metopes, supporting an attic with a marble inscription. Between the columns there were niches, hosting statues or war trophies. The arch was built in puddingstone, a sedimentary conglomerate stone typical of the area.

Today it has lost its attic, it was replaced by a slate roof, the niches were partially closed, some ashlars were substituted, part of the trabeation was reintegrated.

Throughout its life, the arch underwent many transformations. The most recent ones are linked to the restoration work carried out by Alfredo D'Andrade between 1883 and 1891 (a very wellknown intervention in the history of restoration) and by the Egyptologist Ernesto Schiapparelli in 1912-14. After his intervention, which was very extensive, during the Second World War the arch was protected with wood scaffoldings.

Until 1961 the road crossed under the arch vault, causing an increasing exposition of the monument to pollution. To solve this problem a roundabout was built around the arch.

Anyway, to monitor and to preserve the health of its monument, the Superintendency of Aosta, together with its inner laboratory of analysis, RAVA, carried out an intensive monitoring and control activity on the building, collecting a lot of analytical and statistical data that now constitute an indispensable asset for the drafting of the restoration project.

All this richness of data, archival documentation, analysis, tests needed to be made easily accessible by the professionals in charge of the conservation project. The HBIM approach is the most promising system for managing three-dimensional data and, although it faces some difficulties in modelling, it is also the most suitable tool for linking geometric and thematic data. The studies, mainly done by Aosta Superintendency Laboratory for Scientific analysis, produced an important collection of historical studies, chemical and physical characterization of materials and pathologies, studies of the conservation state and risk factors (Appolonia et al., 2007) and microclimatic and environmental condition analyses and records (Ponziani, 2008) (Semprini et al., 2007) (Ponziani, Appolonia et al., 2012).

RAVA is not interested, for the time being, in a structural analysis of the monument, diagnostic campaign aimed to investigate the mechanical property of the materials has never been done, nor other techniques (radar, sonic surveys) aimed to understand the inner structure of the monument.

For these reasons the modelling is limited to the surface, the thickness of the ashlars has been approximated with empirical observation during the modelling phase, mainly on the dimension the ashlar should have to accommodate the angular elements and provide support for the upper level.

\section{RESULTS}

\subsection{Survey}

It was decided to carry out an accurate survey, the fundamental basis for the construction of the HBIM model. The survey, carried out with an integrated approach, has been done with the use of terrestrial laser scanner (Leica RTC360), ground photogrammetry (Canon Eos $5 \mathrm{~d}$ with $35 \mathrm{~mm}$ lens) and drone (Dji Mavic 2 pro), both georeferenced into a local topographic network.

The final result of the survey, the point cloud of Figure 1, integrates outcomes of ground laser scanner point cloud and drone photogrammetric cloud and it is the starting point of this research. The point cloud is characterised by colour thanks to image acquisition of the drone and the presence of a digital camera on the scanner.

When imported into the BIM Authoring software, Autodesk Revit is used in this research, the point cloud is very easy to use and to manage. According to the visualisation tools of Autodesk Revit, it can be sliced, moved, rotated. But it also keeps the colour values for each point, so it can be seen also in a realistic view (as far as allowed by the quality of colours).

\subsection{Federated models}

The survey model is the point cloud, inserted directly into the BIM software Autodesk Revit, and it is the geometric reference, accurate and with the possibility of queries concerning all metric information.

The conceptual model, instead, is the one that has been realized choosing as elements of maximum granularity - the stone ashlar- that build the arch; their dimension allowed such type of modelling, without resulting in an unmanageable number of elements. Choosing the ashlars as elements of maximum granularity allows referencing both graphical, numerical and written information directly on a $3 \mathrm{D}$ object. 

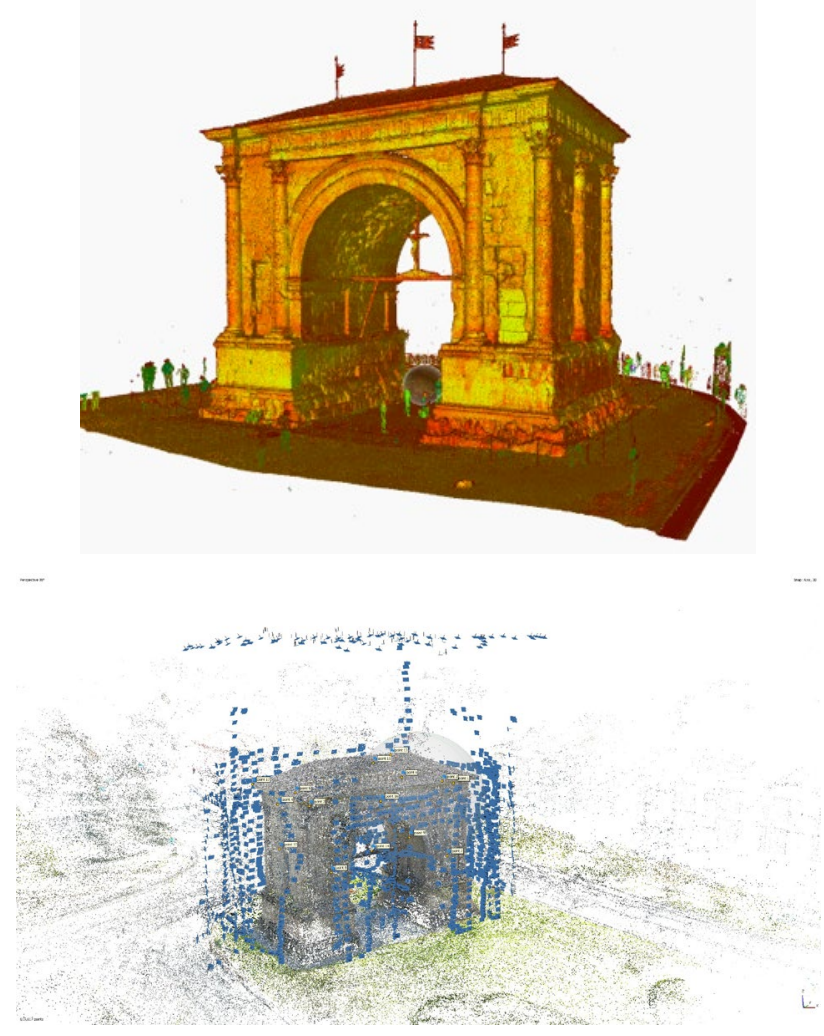

Figure 1: Point cloud of the Arch, from laser scanner (top), from UAV photogrammetry (bottom)

In this way, the information regarding the real shape of the elements should be carried only by the survey model, while Conceptual model's elements have the role of information carrier. To successfully carry the information is sufficient to have an object in its correct position (and topological relationship with its context), hence their shape and decoration could be simplified, providing that the real elements can be still recognized.

\subsection{Conceptual Model construction}

This first operation of segmentation of the monument - not of the model - has traced the construction techniques implemented to build the Arch which provided a limited number of elements that were then finished on-site and took on specific forms. These forms have been made hard to recognize due to the extensive decays. Instead of simplifying irregular shapes, to help the recognition of the architectonic element the choice was to recreate the original ashlar shape as if it were new.

This was done through the analysis of the three-dimensional models coming from the survey (point cloud sections and orthophotos) of the representations in literature, the geometric rules for the construction of the architectural orders and of the comparative studies (other arches of the same period, of the same geographical context) and so the original shape of the ashlars have been identified.

Revit labels the building objects by Family, Category, Type and Instance. Families can be System Families, Loadable and In Place Families. Families are collected in Categories, corresponding to the built elements, such as walls, beams, columns, floors, windows, railings, roofs, doors, etc, those
Categories include all the families, System, Loadable, and In Place.

With the Arch of Augustus it was not possible to use System families. Pre-set elements, such as Walls, pilasters, or beams, follow modern constructions schemes, they connect automatically on what they believe is logical. For example, a beam on a pilaster is jointed, two walls which lay on the same plane and have the same height are automatically merged into one, this behaviour cannot be neglected, since is important for us that the single elements stay distinguished.

The better choice for building the ashlars was to use Loadable families, the category was called 'generic model' since no existing category could match our elements. Generic models are not the best category to use, even if the ashlars were divided into multiple Types, they all belong to the same Category.

Filtering works by Categories, so is not possible to filter specific elements (such as columns, or angular elements) from a general selection, because it will only display a certain number of Generic Models. In the end, the issues of using generic models were smaller than the issues that would have come by using pre-set library or other complex procedures, so it was decided to follow this path.

For each ashlar, a parametric family was built, using the profiles and shapes obtained comparing the geometric rules of the architectural orders with point cloud sections. With parametric families we do not have to create a solid for each single ashlar present on the monument, is sufficient to identify a prototype for each variation (Types) of the ashlars, create the threedimensional object (Family), and then place an Instance in the model space, adapting the dimension to the real object read on the Survey model (Figure 2)

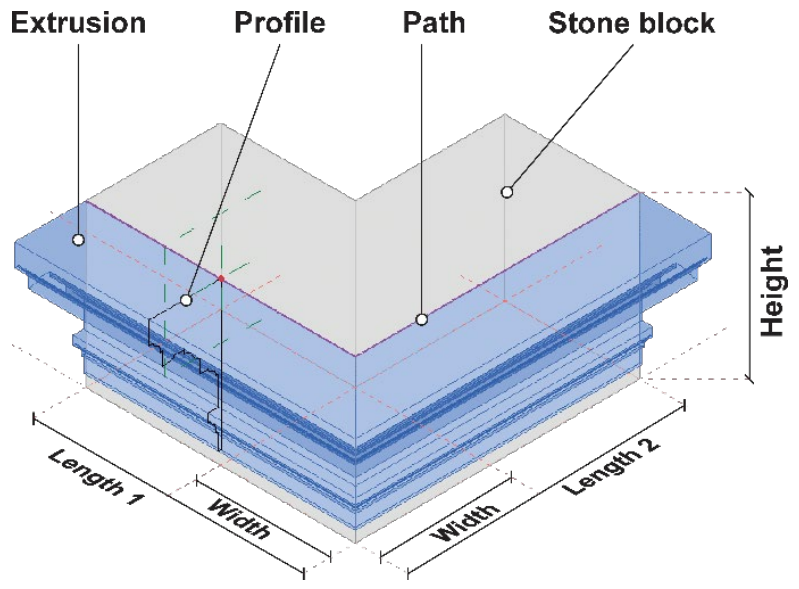

Figure 2: Anatomy of an angular parametric ashlar

The process resulted in an abacus of ashlars, (such as the parallelepiped "generic ashlar"the "angular ashlars", the "column base", "column body", "capital ashlar", "entablature ashlar") each with its own characteristics, but above all with parametric geometric features and specific functions which stills allows the recognition of the architectural elements.

With less than fifty elements it has been possible to build the final model containing more than 1500 ashlars. (Figure 3) 


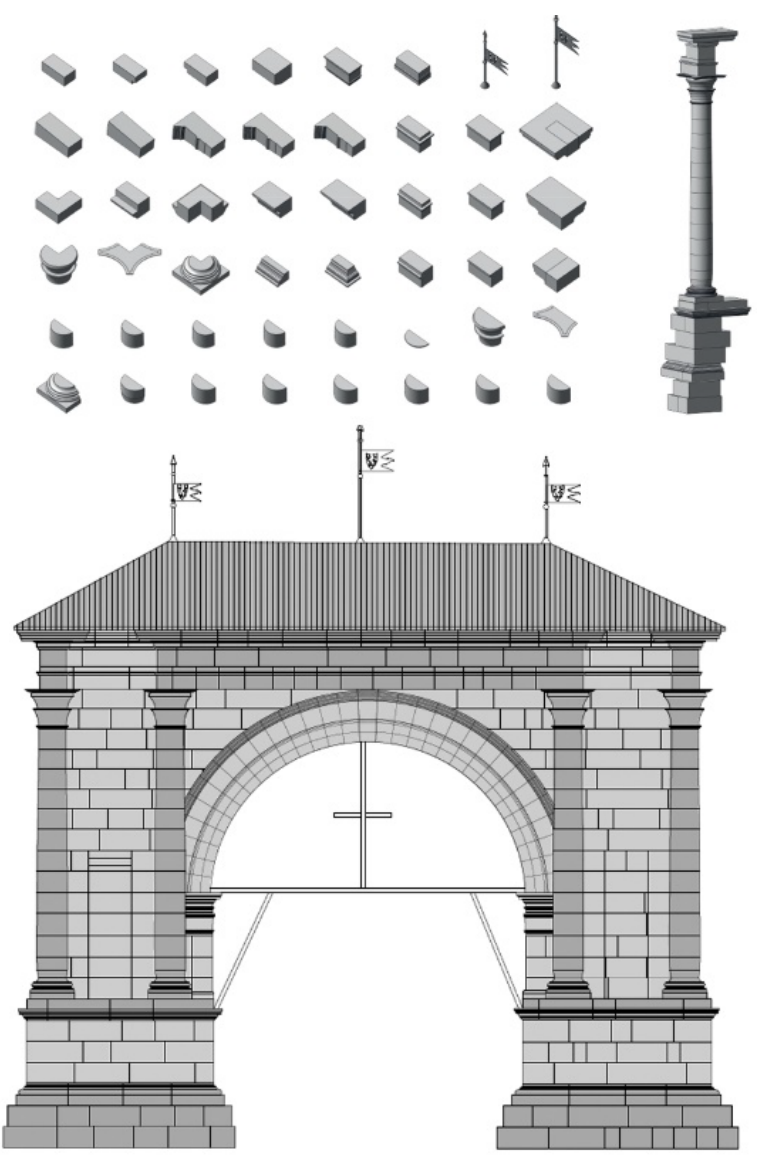

Figure 3: (top) All of the geometric families of ashlars, (bottom) west elevation with the evidence of each single element.

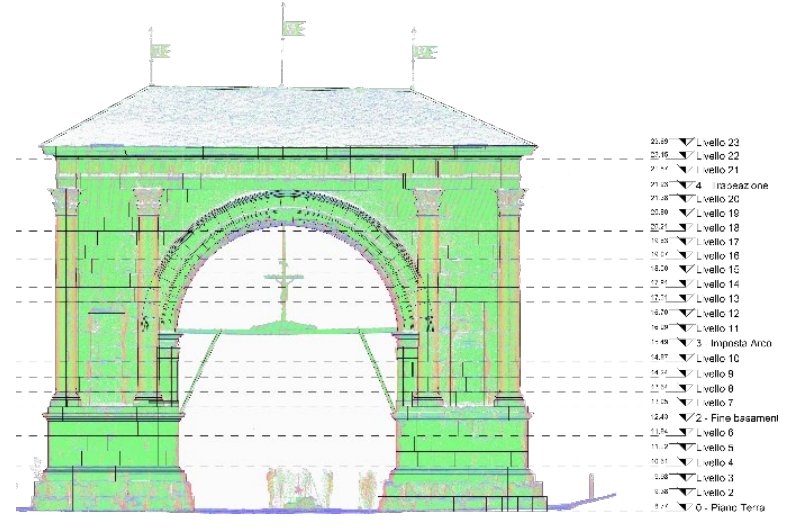

Figure 4: West elevation with the point cloud normal visualization and the levels

\subsection{Information linked to the ashlar}

Inside Autodesk Revit each three-dimensional element lays on a reference plane, these planes are called Levels; being the ashlar laid in horizontal rows, each ashlar is automatically connected to a level, the construction techniques gives us already one way to indexing and querying the model, sorting the stone blocks by levels, unique ID are given automatically by the software, but additional ID with codified meaning can be added. (Figure 4)
Each ashlar was then assigned historical information (interventions undergone), conservative information (state of preservation and alteration), risk assessments (exposure to weathering, exposure to erosion), sampled stone blocks: this information is essentially related to the position but not to the individual dimensions of the object (Figure 5). As we can see neither of these views are heavily affected by the idealization and simplification of the real shapes.

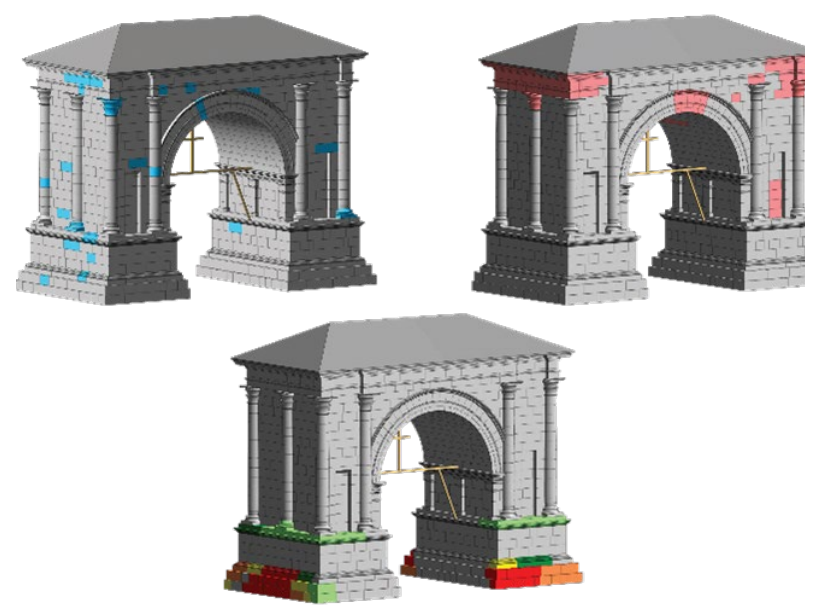

Figure 5: In the different filtered representations, colors are connected to Sampled Ashlars (top left), restored ashlars(top right), risk assestment (bottom).

\subsection{Additional information}

Other information can be hypothesized by comparing the survey model (as-built) with the conceptual ones, (which reconstruct the original shape of the ashlar) to show more information (Figure 6).

The representation, made inside Autodesk Revit with the application As-Built, cannot be considered only as an accuracy map to give a representation of the quality of the modelling step. It is well known that the modelling stage had no goal of accuracy.

On the contrary, this colour map is new information that described the parts that are more deteriorated, because of the erosion and the loss of material.

Not all of the information is written on the single stone blocks, some data are linked directly to the model such as the bidimensional .dwg files containing the regional and municipal cartography. The Survey model itself, being the point cloud linked in the model space, contains information about the immediate surrounding of the building, the site and terrain slopes, presence of corbels, trees, urban furniture and aerials systems, all useful information, when planning the worksite accessibility and provisional works design.

\subsection{Use of federated models and BIM tools}

Following this approach but being very careful not to lose the position information of each single segment, we obtained two georeferenced models in the same reference system (and in the same model space): one useful to extract metrical information (survey model) and one useful for the management of all information (conceptual model). Architects, restorers, designers, users of the BIM system, then have the choice to choose, among 
the federated models, the one of greatest interest for their own activities, both documentary and design.

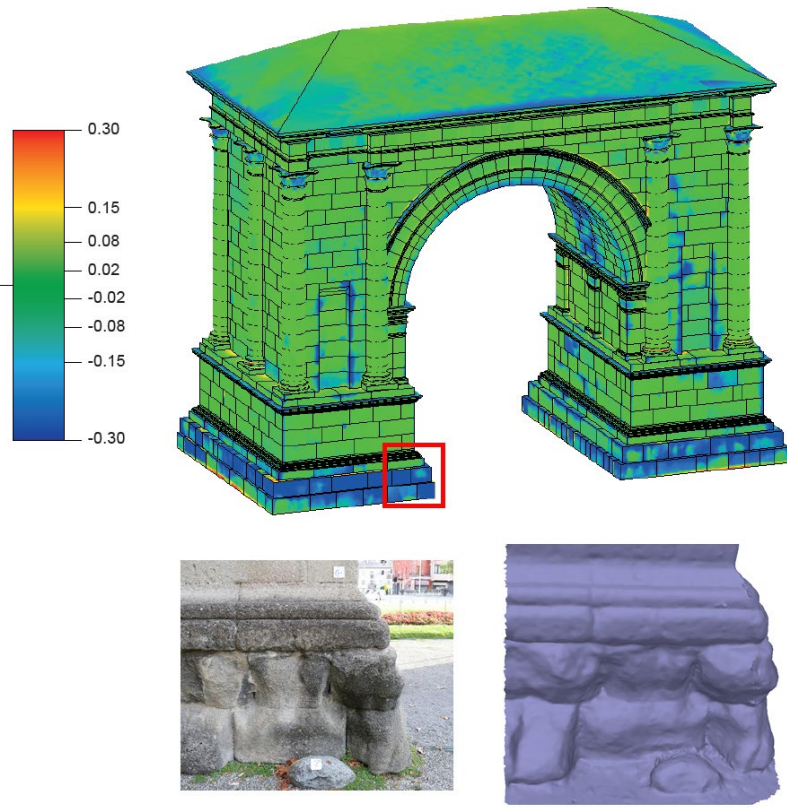

Figure 6: (top) Comparison between the survey model and the conceptual one. The biggest differences are represented in blue. The red box (bottom) localizes the basement of the arch where the effects of decay are evident. They can be recognized in the picture (on the left) or in the mesh model (on the right).

Having a conceptual model allows us to use some of the BIM tools available with the software chosen, among them the possibility to extract quantity takeoff, i.e. for a cost estimation. Not having reliable information beyond the surface, we can only extract quantities related to the surface area of the ashlars, which is a calculated parameter. Using idealized shapes the area will suffer a certain degree of inaccuracy if compared with the surface area measured on the point cloud or on the photogrammetric mesh. This is a minor issue since cost evaluation based on the unit surface are generally calculated based on bi-dimensional drawings, then coefficients are applied to consider the tridimensionality of the object. These coefficients, properly corrected, can be applied also for cost evaluation extracted from the model.

Another tool (which can not work with point clouds only) that comes with Revit is the sun path simulation engine, which allows designers and restorers to better study the shadow areas on the monument, which influence water behaviour on the stone.

Clash detections can be done with both conceptual and survey models, for preliminary studies conceptual model will work fine, and when millimetric accuracy is needed we will use the survey model. Automatic clash detection has not been tested for this model, but since the only element that will clash with the monument will be provisional works (the lighting system is placed around the monument and not on it) it will be always possible to create views on the anchoring points and have a "manual" clash detection between the architecture and the provisional works.

\section{CONCLUSIONS}

The concept of the federated model is very typical of BIM approach, but also in the HBIM context, it can play an important role. Especially for all those cases when the modelling stage is particularly complex because of complex shapes, state of conservation, etc. In this paper we stated the importance and usefulness of the federation of survey model and conceptual models. Intended as the $3 \mathrm{D}$ point cloud and its conceptualization through a poarametric BIM model with a specific granularity chosen looking to the building itself.

Another field of application could be related to those design activities that don't imply the necessity of a single very accurate model (preventive planned conservation) but can use different sources for data and information and in the same time make good use of BIM tools.

Continuing along the same path, the next step provides the possibility to attach information directly to the point cloud (or rather to a part of it). Creating a bidirectional relationship between survey model and conceptual model could be another relevant issue: for this, it will be necessary to link the ideal schematic concept to its real portion only. This operation today is still quite complex due to the difficulty of managing many surfaces or point clouds, within the same file.

\section{REFERENCES}

Adami A., 2021, HBIM per i grandi complessi architettonici. In Geomatica e HBIM per i beni culturali, (edited by. A. Adami) Architectural Design and History, FrancoAngeli s.r.l., Milano, pages 34-61.

Adami, A., Appolonia, L., Scala, B., 2021. The Arch of Augustus in Aosta: data and analysis reuse. In Proceedings of the joint international event 9th ARQUEOLÓGICA 2.0 \& 3rd GEORES, Valencia (Spain). 26-28 April 2021 CGAL, 2021. Computational Geometry Algorithms Library, http://www.cgal.org (Last accessed: Oct 2021).

Appolonia, L., Migliorini, S., Idone, A., Piccirillo, A., 2007. L'Arco di Augusto in Aosta: un esempio di percorso programmato tra progettazione e diagnostica. In Bollettino della Soprintendenza per i beni e le attività culturali, 4, pp. 215-228

Banfi, F., 2019. The integration of a scan-to-HBIM process in BIM application: the development of an add-in to guide users in Autodesk Revit, In. Int. Arch. Photogramm. Remote Sens. Spatial Inf. Sci., XLII-2/W11, 141-148, https://doi.org/10.5194/isprs-archives-XLII-2-W11-141-2019, 2019.

Barazzetti L., 2016. Parametric as-built model generation of complex shapes from point clouds in Advanced Engineering Informatics, Volume 30, Issue 3, 2016, Pages 298-311, ISSN 1474-0346, https://doi.org/10.1016/j.aei.2016.03.005.

R. Brumana - D. Oreni - L. Barazzetti - B. Cuca · M. Previtali · F. Banfi, 2020, Survey and Scan to BIM Model for the Knowledge of Built Heritage and the Management of Conservation Activities in Digital Transformation of the Design, Construction and Management Processes of the Built Environment, Research for Development, https://doi.org/10.1007/978-3-030-33570-0_35.

Borghi A., 2017, Le Pietre ornamentali storiche e contemporanee della Città di Aosta, in Atti del convegno 
nazionale geologia urbana di Aosta, Geologia dell'Ambiente, supplemento al $\mathrm{n}^{\circ}$ 2/2017, pp- 34-40

Costantino, D., Pepe, M., Restuccia, A.G., 2021. Scan-to-HBIM for Conservation and Preservation of Cultural Heritage Building: The Case Study of San Nicola in Montedoro Church (Italy). Applied Geomatics, 2021.

https://doi.org/10.1007/s12518-021-00359-2

Idone A., 2007, L'arco di augusto in Aosta: un esempio di percorso programmato tra progettazione e diagnostica. Master Degree Thesis in Scienza e Tecnologia per i Beni Culturali, università degli studi di Torino Facoltà di scienze Matematiche Fisiche e Maturali.

Ponziani D., 2008, L'Arco di Augusto in Aosta: un esempio di monitoraggio microclimatico finalizzato alla conservazione, Master Thesis in Materiali e Tecniche diagnostiche nel settore dei Beni Culturali dell'Università di Pisa.

Ponziani D., Ferrero E., Appolonia L., Migliorini S., 2012, Effects of temperature and humidity excursion and wind exposure on the Arch of Augustus in Aosta, in Journal of Cultural Heritage, Vol. 13 No. 4.

Santagati, C., Papacharalambous, D., Sanfilippo, G., Bakirtzis, N., Laurini, C., Hermon, S.,2021. HBIM Approach for the Knowledge and Documentation of the St. John the Theologian Cathedral in Nicosia (Cyprus). J. Archaeol. Sci. Rep. 2021, 36, 102804

Semprini P., Appolonia L., Furlani F. et al., 2007, Applicazione di un codice di fluidodinamica computazionale (CFD) a studi microclimatici su beni culturali in ambienti aperti, in $V I$ Congresso Nazionale IGIIC - Lo stato dell'Arte programmato tra progettazione e diagnostica. In Bollettino della Soprintendenza per i beni e le attività culturali, 4, pp. 215-228

Solihin, W., Eastman, C., Cheol Lee, Y., 2016. A framework for fully integrated building information models in a federated environment, Advanced Engineering Informatics, Volume 30, Issue 2, 2016, Pages 168-189, ISSN 1474-0346, https://doi.org/10.1016/j.aei.2016.02.007

Yang, X.; Grussenmeyer, P.; Koehl, M.; Macher, H.; Murtiyoso, A.; Landes, T. Review of Built Heritage Modelling: Integration of HBIM and Other Information Techniques. J. Cult. Herit. 2020, 46, 350-360. 